\title{
A New Solution Operator of One-Dimensional $p$-Laplacian with a Sign-Changing Weight and Its Application
}

\author{
Inbo Sim ${ }^{1}$ and Yong Hoon Lee ${ }^{2}$ \\ ${ }^{1}$ Department of Mathematics, University of Ulsan, Ulsan 680-749, Republic of Korea \\ 2 Department of Mathematics, Pusan National University, Busan 609-735, Republic of Korea \\ Correspondence should be addressed to Yong Hoon Lee, yhlee@pusan.ac.kr
}

Received 22 February 2012; Accepted 7 May 2012

Academic Editor: Ferhan M. Atici

Copyright (c) 2012 I. Sim and Y. H. Lee. This is an open access article distributed under the Creative Commons Attribution License, which permits unrestricted use, distribution, and reproduction in any medium, provided the original work is properly cited.

We establish a new solution operator for the following problem $-\varphi_{p}\left(u^{\prime}\right)^{\prime}=g, t \in(0,1), u(0)=0=$ $u(1)$, where $\varphi_{p}(x)=|x|^{p-2} x, p>1$. $g$ may be singular at the boundary or change signs or may not be in $L^{1}(0,1)$ so that this solution operator can cover larger class of $g$ than previously known ones. As an application, by checking complete continuity of the solution operator, we show the existence of nontrivial solutions for $p$-Laplacian $\varphi_{p}\left(u^{\prime}\right)^{\prime}+\lambda h(t) f(u(t))=0, t \in(0,1), u(0)=0=u(1)$, where $\lambda>0$ a parameter and $f \in C(\mathbb{R}, \mathbb{R})$ and $f(0)>0$ and $h$ may change signs or may be beyond of $L^{1}(0,1)$.

\section{Introduction}

The solution operator approach has been used to show the existence of solutions for wide class of nonlinear differential equations. As it is wellknown, a solution operator plays a key role when we employ nonlinear analytic methods such as degree theory, fixed point index theory, and bifurcation theory. In this paper, we are concerned with a solution operator for one-dimensional $p$-Laplacian with a sign-changing singular weight.

Let us summarize a brief history about solution operators for one-dimensional $p$ Laplacian with Dirichlet boundary condition

$$
\begin{gathered}
-\varphi_{p}\left(u^{\prime}\right)^{\prime}=g, \quad t \in(0,1), \\
u(0)=0=u(1),
\end{gathered}
$$

where $\varphi_{p}(x)=|x|^{p-2} x, p>1$, and $g \in L_{\mathrm{loc}}^{1}(0,1)$. 
First, when $g \in L^{1}(0,1)$, a solution operator was introduced by Manásevich and Mawhin $[1,2]$. In this case, we know that all solutions of $(A)$ are in $C^{1}[0,1]$ so that by a solution of problem $(A)$, we understand a function $u \in C^{1}[0,1]$ with $\varphi_{p}\left(u^{\prime}\right)$ absolutely continuous which satisfies $(A)$. Key step of establishing a solution operator for this case is to show that, for each $g \in L^{1}(0,1)$, the equation

$$
\int_{0}^{1} \varphi_{p}^{-1}\left(a(g)+\int_{0}^{s} g(\tau) d \tau\right) d t=0
$$

has a unique solution $a=a(g)$ where $a: L^{1}(0,1) \rightarrow \mathbb{R}$ is a continuous function. Defining

$$
G(g)(t) \triangleq \int_{0}^{t} \varphi_{p}^{-1}\left(a(g)+\int_{0}^{s} g(\tau) d \tau\right) d s
$$

problem $(A)$ can be equivalently written as $u=G(g)$.

Secondly, when $g \geq 0$ and satisfies

$$
\int_{0}^{1 / 2} \varphi_{p}^{-1}\left(\int_{s}^{1 / 2} g(\tau) d \tau\right) d s+\int_{1 / 2}^{1} \varphi_{p}^{-1}\left(\int_{1 / 2}^{s} g(\tau) d \tau\right) d s<\infty
$$

a solution operator was studied by Agarwal et al. [3]. We note that a function satisfying (1.3) needs not be in $L^{1}(0,1)$. In this case, we know that solutions of $(A)$ may not be in $C^{1}[0,1]$ so that, by a solution of problem $(A)$, we understand a function $u \in C[0,1] \cap C^{1}(0,1)$ with $\varphi_{p}\left(u^{\prime}\right)$ absolutely continuous which satisfies $(A)$. Define a function $x$ by

$$
x(t)=\int_{0}^{t} \varphi_{p}^{-1}\left(\int_{s}^{t} g(\tau) d \tau\right) d s-\int_{t}^{1} \varphi_{p}^{-1}\left(\int_{t}^{s} g(\tau) d \tau\right) d s
$$

for $0<t<1$. It is easy to see that $x$ is continuous, strictly increasing in $(0,1)$ and $x\left(0^{+}\right)<0<$ $x\left(1^{-}\right)$. Thus, $x$ has a unique zero in $(0,1)$, say $A_{g}$. Then, we see that

$$
\int_{0}^{A_{g}} \varphi_{p}^{-1}\left(\int_{s}^{A_{g}} g(\tau) d \tau\right) d s=\int_{A_{g}}^{1} \varphi_{p}^{-1}\left(\int_{A_{g}}^{s} g(\tau) d \tau\right) d s
$$

and defining

$$
G(g)(t) \triangleq \begin{cases}\int_{0}^{t} \varphi_{p}^{-1}\left(\int_{s}^{A_{g}} g(\tau) d \tau\right) d s, & 0 \leq t \leq A_{g}, \\ \int_{t}^{1} \varphi_{p}^{-1}\left(\int_{A_{g}}^{s} g(\tau) d \tau\right) d s, & A_{g} \leq t \leq 1,\end{cases}
$$

problem $(A)$ can be equivalently written as $u=G(g)$. 
Now let us consider the case that $g$ may change signs and belongs to $\mathscr{H} \subset L_{\text {loc }}^{1}(0,1)$ which is defined as

$$
\mathscr{H}=\left\{g: \int_{0}^{1 / 2} \varphi_{p}^{-1}\left(\int_{s}^{1 / 2}|g(\tau)| d \tau\right) d s+\int_{1 / 2}^{1} \varphi_{p}^{-1}\left(\int_{1 / 2}^{s}|g(\tau)| d \tau\right) d s<\infty\right\} .
$$

Defining $g:(0,1) \rightarrow \mathbb{R}$ by

$$
g(t)= \begin{cases}t^{-\alpha}, & 0<t \leq \frac{1}{2} \\ -1, & \frac{1}{2}<t<1\end{cases}
$$

where $1<\alpha<p$, it is not hard to check that $g \in \mathscr{l}$, but $g \notin L^{1}(0,1)$.

For $g \in \mathscr{t}$ but $g \notin L^{1}(0,1)$, existence of solution for (1.1) is not known so that Manásevich-Mawhin's approach cannot be applied directly. For $g$ sign changing, the uniqueness of zeros of function $x$ in (1.4) is not guaranteed so that Agarwal et al.'s approach cannot be applied directly either. The aim of this paper is to introduce a new solution operator for $(A)$ under this situation.

As an application, we will make use of newly established solution operator to show the existence of nontrivial solutions with respect to a parameter for the following type of nonlinear problem

$$
\begin{gathered}
\varphi_{p}\left(u^{\prime}\right)^{\prime}+\lambda h(t) f(u(t))=0, \quad t \in(0,1), \\
u(0)=0=u(1),
\end{gathered}
$$

where $\lambda>0$ a parameter, $h \in \mathscr{L}, f \in C(\mathbb{R}, \mathbb{R})$, and $f(0)>0$.

This kind of problems can be classified as cases $f(0)>0, f(0)=0$, or $f(0)<0$, and, under additional nonlinear growth conditions, it is easy to see that bifurcation phenomena usually happen for the case of $f(0)>0$. In this paper, we will employ the global continuation theorem to get an existence result. One of the major steps for proof is to show the complete continuity of corresponding solution operator for $\left(P_{\lambda}\right)$. There have been many studies for $f$ nonnegative with $f(0)>0$ and $h \geq 0$. Many authors are interested in finding positive solutions mainly employing the fixed-point index theory on a cone. Since $h$ may change signs in our case, this method is not suitable. Hai [4] investigated semilinear boundary value problems with a sign-changing weight; however, his method is also restricted to fit our quasilinear case.

The paper is organized as follow. In Section 2, we establish a solution operator for $(A)$, when $g \in \mathscr{H}$. In Section 3, we prove the continuity and compactness of the solution operator for problem $\left(P_{\curlywedge}\right)$. In Section 4, we obtain the existence of non-trivial solutions for $\left(P_{\curlywedge}\right)$.

\section{A Solution Operator}

In this section, we construct a new solution operator for $(A)$.

Theorem 2.1. For each $g \in \mathscr{d}$, there is a unique solution $u \in C[0,1] \cap C^{1}(0,1)$ for $(A)$. 
The proof follows the next two lemmas. We first show the existence of a solution for $(A)$.

Lemma 2.2. For each $g \in \mathscr{d}$, there is a unique constant $\alpha=\alpha(g) \in \mathbb{R}$ such that a function $u$ defined by

$$
u(t)= \begin{cases}\int_{0}^{t} \varphi_{p}^{-1}\left(\alpha+\int_{s}^{1 / 2} g(r) d r\right) d s, & 0 \leq t \leq \frac{1}{2} \\ \int_{t}^{1} \varphi_{p}^{-1}\left(-\alpha+\int_{1 / 2}^{s} g(r) d r\right) d s, & \frac{1}{2} \leq t \leq 1\end{cases}
$$

satisfies $u \in C[0,1] \cap C^{1}(0,1)$ and $u$ solves $(A)$. Furthermore, we have

$$
\int_{0}^{1 / 2} \varphi_{p}^{-1}\left(\alpha+\int_{s}^{1 / 2} g(r) d r\right) d s=\int_{1 / 2}^{1} \varphi_{p}^{-1}\left(-\alpha+\int_{1 / 2}^{s} g(r) d r\right) d s .
$$

Remark 2.3. For $a, b>0$,

$$
\varphi_{p}^{-1}(a+b)=(a+b)^{1 /(p-1)} \leq \begin{cases}a^{1 /(p-1)}+b^{1 /(p-1)}, & p>2 \\ 2^{(2-p) /(p-1)}\left(a^{1 /(p-1)}+b^{1 /(p-1)}\right), & 1<p \leq 2 .\end{cases}
$$

Thus for $0<t \leq 1 / 2$,

$$
|u(t)| \leq C_{p}\left(\int_{0}^{t}|\alpha|^{1 /(p-1)} d t+\int_{0}^{t} \varphi_{p}^{-1}\left(\int_{s}^{1 / 2}|g(r)| d r\right) d s\right)<\infty,
$$

where

$$
C_{p}= \begin{cases}1, & p>2 \\ 2^{(2-p) /(p-1)}, & 1<p \leq 2 .\end{cases}
$$

Similarly, we get an upper bound of $|u|$ on $(1 / 2,1)$. It is clear that $u(0)=0=u(1)$.

Proof of Lemma 2.2. Define

$$
\begin{array}{ll}
v_{\alpha}(t) \triangleq \int_{0}^{t} \varphi_{p}^{-1}\left(\alpha+\int_{s}^{1 / 2} g(r) d r\right) d s, & 0 \leq t \leq \frac{1}{2}, \\
w_{\alpha}(t) \triangleq \int_{t}^{1} \varphi_{p}^{-1}\left(-\alpha+\int_{1 / 2}^{s} g(r) d r\right) d s, & \frac{1}{2} \leq t \leq 1 .
\end{array}
$$

Then, by Lebesgue dominated convergence theorem, we see that $v_{\alpha}(1 / 2)$ and $w_{\alpha}(1 / 2)$ are continuous on $\alpha$. Because of the strict monotonicity of $\varphi_{p}$, it is easy to show that $v_{\alpha}(1 / 2)$ is strictly increasing on $\alpha$ and $w_{\alpha}(1 / 2)$ is strictly decreasing on $\alpha$. 
We claim that $v_{\alpha}(1 / 2) \rightarrow \infty, w_{\alpha}(1 / 2) \rightarrow-\infty$ as $\alpha \rightarrow \infty$, and $v_{\alpha}(1 / 2) \rightarrow-\infty$, $w_{\alpha}(1 / 2) \rightarrow \infty$ as $\alpha \rightarrow-\infty$. Since $(\alpha / 2)+\int_{s}^{1 / 2} g(r) d r>0$ for $1 / 3 \leq s \leq 1 / 2$, for sufficiently large $\alpha>0$, we have

$$
\begin{aligned}
v_{\alpha}\left(\frac{1}{2}\right) & =\int_{0}^{1 / 2} \varphi_{p}^{-1}\left(\alpha+\int_{s}^{1 / 2} g(r) d r\right) d s \\
& =\int_{0}^{1 / 3} \varphi_{p}^{-1}\left(\alpha+\int_{s}^{1 / 2} g(r) d r\right) d s+\int_{1 / 3}^{1 / 2} \varphi_{p}^{-1}\left(\alpha+\int_{s}^{1 / 2} g(r) d r\right) d s \\
& \geq \frac{1}{6}\left(\frac{\alpha}{2}\right)^{1 /(p-1)}+\int_{0}^{1 / 3} \varphi_{p}^{-1}\left(\int_{s}^{1 / 2} g(r) d r\right) d s \\
& \geq \frac{1}{6}\left(\frac{\alpha}{2}\right)^{1 /(p-1)}-\int_{0}^{1 / 2} \varphi_{p}^{-1}\left(\int_{s}^{1 / 2}|g(r)| d r\right) d s \rightarrow \infty
\end{aligned}
$$

as $\alpha \rightarrow \infty$. Since $-\alpha / 2+\int_{1 / 2}^{s} g(r) d r<0$ for $1 / 2 \leq s \leq 2 / 3$, for sufficiently large $\alpha>0$, we have

$$
\begin{aligned}
w_{\alpha}\left(\frac{1}{2}\right) & =\int_{1 / 2}^{1} \varphi_{p}^{-1}\left(-\alpha+\int_{1 / 2}^{s} g(r) d r\right) d s \\
& =\int_{1 / 2}^{2 / 3} \varphi_{p}^{-1}\left(-\alpha+\int_{1 / 2}^{s} g(r) d r\right) d s+\int_{2 / 3}^{1} \varphi_{p}^{-1}\left(-\alpha+\int_{1 / 2}^{s} g(r) d r\right) d s \\
& \leq-\frac{1}{6}\left(\frac{\alpha}{2}\right)^{1 /(p-1)}+\int_{2 / 3}^{1} \varphi_{p}^{-1}\left(\int_{1 / 2}^{s} g(r) d r\right) d s \\
& \leq-\frac{1}{6}\left(\frac{\alpha}{2}\right)^{1 /(p-1)}+\int_{1 / 2}^{1} \varphi_{p}^{-1}\left(\int_{1 / 2}^{s}|g(r)| d r\right) d s \rightarrow-\infty,
\end{aligned}
$$

as $\alpha \rightarrow \infty$. Similarly, since $\alpha / 2+\int_{s}^{1 / 2} g(r) d r<0$ for $1 / 3 \leq s \leq 1 / 2$, for negatively large $\alpha<0$, we have

$$
\begin{aligned}
v_{\alpha}\left(\frac{1}{2}\right) & =\int_{0}^{1 / 2} \varphi_{p}^{-1}\left(\alpha+\int_{s}^{1 / 2} g(r) d r\right) d s \\
& =\int_{0}^{1 / 3} \varphi_{p}^{-1}\left(\alpha+\int_{s}^{1 / 2} g(r) d r\right) d s+\int_{1 / 3}^{1 / 2} \varphi_{p}^{-1}\left(\alpha+\int_{s}^{1 / 2} g(r) d r\right) d s \\
& \leq-\frac{1}{6}\left(\frac{-\alpha}{2}\right)^{1 /(p-1)}+\int_{0}^{1 / 3} \varphi_{p}^{-1}\left(\int_{s}^{1 / 2} g(r) d r\right) d s \\
& \leq-\frac{1}{6}\left(\frac{-\alpha}{2}\right)^{1 /(p-1)}+\int_{0}^{1 / 2} \varphi_{p}^{-1}\left(\int_{s}^{1 / 2}|g(r)| d r\right) d s \rightarrow-\infty,
\end{aligned}
$$


as $\alpha \rightarrow-\infty$. Since $-\alpha / 2+\int_{1 / 2}^{s} g(r) d r>0$ for $1 / 2 \leq s \leq 2 / 3$, for negatively large $\alpha<0$, we have

$$
\begin{aligned}
w_{\alpha}\left(\frac{1}{2}\right) & =\int_{1 / 2}^{1} \varphi_{p}^{-1}\left(-\alpha+\int_{1 / 2}^{s} g(r) d r\right) d s \\
& =\int_{1 / 2}^{2 / 3} \varphi_{p}^{-1}\left(-\alpha+\int_{1 / 2}^{s} g(r) d r\right) d s+\int_{2 / 3}^{1} \varphi_{p}^{-1}\left(-\alpha+\int_{1 / 2}^{s} g(r) d r\right) d s \\
& \geq \frac{1}{6}\left(\frac{-\alpha}{2}\right)^{1 /(p-1)}+\int_{2 / 3}^{1} \varphi_{p}^{-1}\left(\int_{1 / 2}^{s} g(r) d r\right) d s \\
& \geq \frac{1}{6}\left(\frac{-\alpha}{2}\right)^{1 /(p-1)}-\int_{1 / 2}^{1} \varphi_{p}^{-1}\left(\int_{1 / 2}^{s}|g(r)| d r\right) d s \rightarrow \infty
\end{aligned}
$$

as $\alpha \rightarrow-\infty$. Thus, by intermediate value theorem, there is a unique $\alpha_{0} \in \mathbb{R}$ such that $v_{\alpha_{0}}(1 / 2)=w_{\alpha_{0}}(1 / 2)$. Put

$$
u(t)= \begin{cases}v_{\alpha_{0}}(t), & 0 \leq t \leq \frac{1}{2} \\ w_{\alpha_{0}}(t), & \frac{1}{2} \leq t \leq 1\end{cases}
$$

Then, $u \in C[0,1] \cap C^{1}(0,1)$ and $u$ solves $(A)$.

From the uniqueness of $\alpha$, the following is obvious.

Corollary 2.4. Suppose that $\alpha$ is a function defined in Lemma 2.2. Then, $\alpha$ has the following property:

$$
\alpha(\lambda g)=\lambda \alpha(g)
$$

for all $\lambda \in \mathbb{R}$.

The following lemma guarantees the uniqueness of solutions for $(A)$.

Lemma 2.5. Assume that, for $i=1,2, u_{i} \in C[0,1] \cap C^{1}(0,1)$ satisfies

$$
\begin{gathered}
-\varphi_{p}\left(u^{\prime}\right)^{\prime}=g_{i}, \quad t \in(0,1), \\
u(0)=0=u(1) .
\end{gathered}
$$

If $g_{1} \leq g_{2}$ or $g_{2} \leq g_{1}$ on $[0,1]$, then $u_{1} \leq u_{2}$ or $u_{2} \leq u_{1}$ on $[0,1]$, respectively.

Proof. We only prove the first case. We may use similar argument to show the second case. Let $u_{i}$ be a solution of $\left(A_{i}\right), i=1,2$, and assume $g_{1} \leq g_{2}$ for $0 \leq t \leq 1$. We want to show $u_{1}(t) \leq u_{2}(t), 0 \leq t \leq 1$. If this is false, then there exists $t_{0} \in(0,1)$ with $u_{1}\left(t_{0}\right)>u_{2}\left(t_{0}\right)$. Hence, there is an interval $(a, b) \subset(0,1)$ with $t_{0} \in(a, b)$ such that $u_{1}(t)>u_{2}(t), a<t<b$ and $u_{1}(a)-u_{2}(a)=u_{1}(b)-u_{2}(b)=0$. Let $m=u_{1}(B)-u_{2}(B)$ be the positive maximum 
of $u_{1}(t)-u_{2}(t)$ on $[a, b]$. Then, $B \in[a, b]$ and $u_{1}^{\prime}(B)=u_{2}^{\prime}(B)$. Integrating both sides $\left(A_{1}\right)$ and $\left(A_{2}\right)$ over $[s, B], a<s<B$, we have

$$
\begin{aligned}
& u_{1}^{\prime}(s)=\varphi_{p}^{-1}\left(\varphi_{p}\left(u_{1}^{\prime}(B)\right)+\int_{s}^{B} g_{1}(r) d r\right) \\
& u_{2}^{\prime}(s)=\varphi_{p}^{-1}\left(\varphi_{p}\left(u_{2}^{\prime}(B)\right)+\int_{s}^{B} g_{2}(r) d r\right) .
\end{aligned}
$$

Integrating both sides of the above equalities over $[a, B]$, we get

$$
\begin{aligned}
& u_{1}(B)-u_{1}(a)=\int_{a}^{B} \varphi_{p}^{-1}\left(\varphi_{p}\left(u_{1}^{\prime}(B)\right)+\int_{s}^{B} g_{1}(r) d r\right) d s, \\
& u_{2}(B)-u_{2}(a)=\int_{a}^{B} \varphi_{p}^{-1}\left(\varphi_{p}\left(u_{2}^{\prime}(B)\right)+\int_{s}^{B} g_{2}(r) d r\right) d s .
\end{aligned}
$$

Thus, we obtain

$$
0<m=u_{1}(B)-u_{2}(B) \leq 0,
$$

which is a contradiction. This completes the proof.

Let us define

$$
G(g)(t)= \begin{cases}\int_{0}^{t} \varphi_{p}^{-1}\left(\alpha+\int_{s}^{1 / 2} g(r) d r\right) d s, & 0 \leq t \leq \frac{1}{2} \\ \int_{t}^{1} \varphi_{p}^{-1}\left(-\alpha+\int_{1 / 2}^{s} g(r) d r\right) d s, & \frac{1}{2} \leq t \leq 1 .\end{cases}
$$

Then, problem $(A)$ can be equivalently written as $u=G(g)$.

\section{Application: Compactness}

In this and the following sections, as an application of new solution operator, we consider a problem of one-dimensional $p$-Laplacian with a singular weight as follows:

$$
\begin{gathered}
\varphi_{p}\left(u^{\prime}\right)^{\prime}+\lambda h(t) f(u(t))=0, \quad t \in(0,1), \\
u(0)=0=u(1),
\end{gathered}
$$

where $\lambda>0$ a parameter, $h \in \mathscr{H}$, and $f \in C(\mathbb{R}, \mathbb{R})$. Note that we do not need any other assumptions on $f$ except the continuity in this section. Employing the solution operator $G$ established in Section 2, we may rewrite $\left(P_{\lambda}\right)$ equivalently as

$$
u=G(\lambda, u)
$$


where $G:(0, \infty) \times C[0,1] \rightarrow C[0,1]$ is defined by

$$
G(\lambda, u)(t) \triangleq \begin{cases}\int_{0}^{t} \varphi_{p}^{-1}\left(\alpha(\lambda h f(u))+\int_{s}^{1 / 2} \lambda h(r) f(u(r)) d r\right) d s, & 0 \leq t \leq \frac{1}{2} \\ \int_{t}^{1} \varphi_{p}^{-1}\left(-\alpha(\lambda h f(u))+\int_{1 / 2}^{s} \lambda h(r) f(u(r)) d r\right) d s, & \frac{1}{2} \leq t \leq 1\end{cases}
$$

The purpose of this section is to show that $G$ is completely continuous on $(0, \infty) \times C[0,1]$. For this, we need to know the properties of function $\alpha:(0, \infty) \times C[0,1] \rightarrow \mathbb{R}$.

Lemma 3.1. $\alpha$ sends bounded sets in $(0, \infty) \times C[0,1]$ into bounded sets in $\mathbb{R}$.

Proof. Assume that $\left\{\lambda_{n}\right\}$ and $\left\{u_{n}\right\}$ are bounded on $(0, \infty)$ and $C[0,1]$, respectively. For convenience, we denote $\alpha_{n}=\alpha\left(\lambda_{n} h f\left(u_{n}\right)\right)$. Suppose that $\left\{\alpha_{n}\right\}$ is unbounded in $\mathbb{R}$. Then, there is a subsequence $\left\{\alpha_{n_{k}}\right\}$ such that $\alpha_{n_{k}} \rightarrow \infty$ or $\alpha_{n_{k}} \rightarrow-\infty$ as $k \rightarrow \infty$. Here, we consider the first case $\alpha_{n_{k}} \rightarrow \infty$. We can prove the second case by the same argument. As in (2.2) of Lemma 2.2, we have

$$
\begin{aligned}
& \int_{0}^{1 / 2} \varphi_{p}^{-1}\left(\alpha_{n_{k}}+\int_{s}^{1 / 2} \lambda_{n_{k}} h(r) f\left(u_{n_{k}}(r)\right) d r\right) d s \\
& =\int_{1 / 2}^{1} \varphi_{p}^{-1}\left(-\alpha_{n_{k}}+\int_{1 / 2}^{s} \lambda_{n_{k}} h(r) f\left(u_{n_{k}}(r)\right) d r\right) d s .
\end{aligned}
$$

As in the proof of Lemma 2.2, we get the following inequality for the left-hand side

$$
\begin{aligned}
0 & \leq \int_{0}^{1 / 2} \varphi_{p}^{-1}\left(\alpha_{n_{k}}-M \int_{s}^{1 / 2}|h(r)| d r\right) d s \\
& \leq \int_{0}^{1 / 2} \varphi_{p}^{-1}\left(\alpha_{n_{k}}+\int_{s}^{1 / 2} \lambda_{n_{k}} h(r) f\left(u_{n_{k}}(r)\right) d r\right) d s
\end{aligned}
$$

for sufficiently large $k$, where $M=\sup \left\{\left|\lambda_{n_{k}}\right| \mid f\left(u_{n_{k}}\right) \|_{\infty}\right\}$. On other hand, as in the proof of Lemma 2.2, we get the following inequality for the right-hand side

$$
\begin{aligned}
& \int_{1 / 2}^{1} \varphi_{p}^{-1}\left(-\alpha_{n_{k}}+\int_{1 / 2}^{s} \lambda_{n_{k}} h(r) f\left(u_{n_{k}}(r)\right) d r\right) d s \\
& \leq \int_{1 / 2}^{1} \varphi_{p}^{-1}\left(-\alpha_{n_{k}}+M \int_{1 / 2}^{s}|h(r)| d r\right) d s<0
\end{aligned}
$$

for sufficiently large $k$, and this is a contradiction.

Lemma 3.2. $\alpha$ is continuous on $(0, \infty) \times C[0,1]$. 
Proof. Assume that $\lambda_{n} \rightarrow \lambda$ in $\mathbb{R}$ and $u_{n} \rightarrow u$ in $C[0,1]$. Then, there exists a constant $M>0$ such that

$$
\left|\lambda_{n}\right|+\left\|u_{n}\right\|_{\infty} \leq M, \quad \forall n
$$

Lemma 3.1 implies that $\left\{\alpha\left(\lambda_{n} h f\left(u_{n}\right)\right)\right\}$ is bounded in $\mathbb{R}$. Hence, it has a convergent subsequence denoted by $\left\{\alpha\left(\lambda_{n_{k}} h f\left(u_{n_{k}}\right)\right)\right\}$. Let

$$
\alpha\left(\lambda_{n_{k}} h f\left(u_{n_{k}}\right)\right) \longrightarrow \widehat{\alpha}
$$

as $k \rightarrow \infty$. As in (2.2) of Lemma 2.2, we have

$$
\begin{aligned}
& \int_{0}^{1 / 2} \varphi_{p}^{-1}\left(\alpha\left(\lambda_{n_{k}} h f\left(u_{n_{k}}\right)\right)+\int_{s}^{1 / 2} \lambda_{n_{k}} h(r) f\left(u_{n_{k}}(r)\right) d r\right) d s \\
& =\int_{1 / 2}^{1} \varphi_{p}^{-1}\left(-\alpha\left(\lambda_{n_{k}} h f\left(u_{n_{k}}\right)\right)+\int_{1 / 2}^{s} \lambda_{n_{k}} h(r) f\left(u_{n_{k}}(r)\right) d r\right) d s .
\end{aligned}
$$

By Lebesgue dominated convergence theorem, as $k \rightarrow \infty$, we get

$$
\int_{0}^{1 / 2} \varphi_{p}^{-1}\left(\widehat{\alpha}+\int_{s}^{1 / 2} \lambda h(r) f(u(r)) d r\right) d s=\int_{1 / 2}^{1} \varphi_{p}^{-1}\left(-\widehat{\alpha}+\int_{1 / 2}^{s} \lambda h(r) f(u(r)) d r\right) d s .
$$

By the uniqueness of $\alpha$, we obtain $\widehat{\alpha}=\alpha(\lambda h f(u))$, and this proves the continuity of $\alpha$.

We have the following corollary using similar argument in the proof of Lemma 3.2.

Corollary 3.3. Let $\lambda_{n} \in(0, \infty)$ satisfy $\lambda_{n} \rightarrow 0$ as $n \rightarrow \infty$. Then, $\alpha\left(\lambda_{n}\right) \rightarrow 0$ as $n \rightarrow \infty$.

Theorem 3.4. $G$ is continuous and compact on $(0, \infty) \times C[0,1]$. 
Proof. First, we show the continuity of $G$. Assume that $\lambda_{n} \rightarrow \lambda$ on $(0, \infty)$ and $u_{n} \rightarrow u$ in $C[0,1]$. Then, from the continuity of $\alpha$ and Lebesgue dominated convergence theorem, we have

$$
\begin{aligned}
\left\|G\left(\lambda_{n}, u_{n}\right)-G(\lambda, u)\right\|_{\infty}= & \sup _{0 \leq t \leq 1}\left|G\left(\lambda_{n}, u_{n}\right)(t)-G(\lambda, u)(t)\right| \\
\leq & \sup _{0 \leq t \leq 1 / 2} \mid \int_{0}^{t} \varphi_{p}^{-1}\left(\alpha\left(\lambda_{n} h f\left(u_{n}\right)\right)+\int_{s}^{1 / 2} \lambda_{n} h(r) f\left(u_{n}(r)\right) d r\right) \\
& \quad-\varphi_{p}^{-1}\left(\alpha(\lambda h f(u))+\int_{s}^{1 / 2} \lambda h(r) f(u(r)) d r\right) d s \mid \\
& +\sup _{1 / 2 \leq t \leq 1} \mid \int_{t}^{1} \varphi_{p}^{-1}\left(\alpha\left(\lambda_{n} h f\left(u_{n}\right)\right)+\int_{1 / 2}^{s} \lambda_{n} h(r) f\left(u_{n}(r)\right) d r\right) \\
& \quad-\varphi_{p}^{-1}\left(\alpha(\lambda h f(u))+\int_{1 / 2}^{s} \lambda h(r) f(u(r)) d r\right) d s \mid \rightarrow 0 .
\end{aligned}
$$

Next, we show that $G$ is uniformly bounded. Let $\mathcal{M}$ be a bounded subset of $(0, \infty) \times$ $C[0,1]$, and let $(\lambda, u) \in \mathcal{M}$, then using the fact that $\alpha$ sends bounded sets on $(0, \infty) \times C[0,1]$ into bounded sets on $\mathbb{R}$ and taking $M=\sup \left\{|\lambda|\|f(u)\|_{\infty}:(\lambda, u) \in \mathcal{M}\right\}$, we obtain

$$
\begin{aligned}
|G(\lambda, u)(t)| & \leq \begin{cases}\int_{0}^{t} \varphi_{p}^{-1}\left(|\alpha(\lambda h f(u))|+M \int_{s}^{1 / 2}|h(r)| d r\right) d s, & 0 \leq t \leq \frac{1}{2}, \\
\int_{t}^{1} \varphi_{p}^{-1}\left(|\alpha(\lambda h f(u))|+M \int_{1 / 2}^{s}|h(r)| d r\right) d s, \quad \frac{1}{2} \leq t \leq 1,\end{cases} \\
& \leq \begin{cases}\int_{0}^{1 / 2} \varphi_{p}^{-1}\left(|K(M, h)|+M \int_{s}^{1 / 2}|h(r)| d r\right) d s<\infty, & 0 \leq t \leq \frac{1}{2} \\
\int_{1 / 2}^{1} \varphi_{p}^{-1}\left(|K(M, h)|+M \int_{1 / 2}^{s}|h(r)| d r\right) d s<\infty, & \frac{1}{2} \leq t \leq 1,\end{cases}
\end{aligned}
$$

where $K(M, h)$ is a constant and the above upper bound is independent of $(\lambda, u) \in \mathcal{M}$.

Finally, we show that $G(\mathcal{M})$ is equicontinuous. By Lemma 3.1, $\{\alpha(\lambda h f(u)):(\lambda, u) \in$ $\mathcal{M}\}$ is bounded so by taking $N=\sup \left\{\varphi_{p}^{-1}(|\alpha(\lambda h f(u))|):(\lambda, u) \in \mathcal{M}\right\}$, we obtain for $0 \leq$ $t_{1}, t_{2} \leq 1 / 2$,

$$
\begin{aligned}
\left|G(\lambda, u)\left(t_{1}\right)-G(\lambda, u)\left(t_{2}\right)\right| & \leq\left|\int_{t_{1}}^{t_{2}} \varphi_{p}^{-1}\left(\alpha(\lambda h f(u))+\int_{s}^{1 / 2} \lambda h(r) f(u(r)) d r\right) d s\right| \\
& \leq C_{p} N\left|t_{2}-t_{1}\right|+C_{p} M \int_{t_{1}}^{t_{2}} \varphi_{p}^{-1}\left(\int_{s}^{1 / 2}|h(r)| d r\right) d s .
\end{aligned}
$$


Since $h \in \mathscr{H}$, the above upper bound tends to 0 as $\left|t_{1}-t_{2}\right|$ does. For case $1 / 2 \leq t_{1}, t_{2} \leq 1$, we may handle similar way. For case $0 \leq t_{1} \leq 1 / 2,1 / 2 \leq t_{2} \leq 1$ (or case $0 \leq t_{2} \leq 1 / 2,1 / 2 \leq t_{1} \leq 1$ ), we can compute the following:

$$
\begin{aligned}
\left|G(\lambda, u)\left(t_{1}\right)-G(\lambda, u)\left(t_{2}\right)\right|= & \mid \int_{0}^{t_{1}} \varphi_{p}^{-1}\left(\alpha(\lambda h f(u))+\int_{s}^{1 / 2} \lambda h(r) f(u(r)) d r\right) d s \\
& -\int_{t_{2}}^{1} \varphi_{p}^{-1}\left(-\alpha(\lambda h f(u))+\int_{1 / 2}^{s} \lambda h(r) f(u(r)) d r\right) d s \mid \\
= & \mid \int_{0}^{1 / 2} \varphi_{p}^{-1}\left(\alpha(\lambda h f(u))+\int_{s}^{1 / 2} \lambda h(r) f(u(r)) d r\right) d s \\
& -\int_{t_{1}}^{1 / 2} \varphi_{p}^{-1}\left(\alpha(\lambda h f(u))+\int_{s}^{1 / 2} \lambda h(r) f(u(r)) d r\right) d s \\
& -\left[\int_{1 / 2}^{1} \varphi_{p}^{-1}\left(-\alpha(\lambda h f(u))+\int_{1 / 2}^{s} \lambda h(r) f(u(r)) d r\right) d s\right. \\
& \left.-\int_{1 / 2}^{t_{2}} \varphi_{p}^{-1}\left(-\alpha(\lambda h f(u))+\int_{1 / 2}^{s} \lambda h(r) f(u(r)) d r\right) d s\right] \mid \\
= & \mid \int_{1 / 2}^{t_{2}} \varphi_{p}^{-1}\left(-\alpha(\lambda h f(u))+\int_{1 / 2}^{s} \lambda h(r) f(u(r)) d r\right) d s \\
& -\int_{t_{1}}^{1 / 2} \varphi_{p}^{-1}\left(\alpha(\lambda h f(u))+\int_{s}^{1 / 2} \lambda h(r) f(u(r)) d r\right) d s \mid \\
\leq & 2 C_{p} \int_{t_{1}}^{t_{2}}\left\{\varphi_{p}^{-1}(|\alpha(\lambda h f(u))|)\right. \\
\leq & 2 C_{p} N\left|t_{2}-t_{1}\right|+2 C_{p} M_{1} \int_{t_{1}}^{t_{2}} \varphi_{p}^{-1}\left(\int_{1 / 2}^{s}|h(r)| d r\right) \\
&
\end{aligned}
$$

where $M_{1}=\varphi_{p}^{-1}(M)$. Thus, by Arzela-Ascoli Theorem, $G$ is compact, and the proof is complete. 


\section{Application: Existence}

In this section, we show the existence of nontrivial solutions for problem $\left(P_{\curlywedge}\right)$ :

$$
\begin{gathered}
\varphi_{p}\left(u^{\prime}\right)^{\prime}+\lambda h(t) f(u(t))=0, \quad t \in(0,1), \\
u(0)=0=u(1),
\end{gathered}
$$

where $\mathcal{\lambda}>0$ a parameter, $h \in \mathfrak{l}$, and $f \in C(\mathbb{R}, \mathbb{R})$. In addition, we assume that

(F) $f(0)>0$ and $f_{\infty} \equiv \lim _{s \rightarrow \infty} f(s) / s^{p-1}=0$.

Based on the solution operator and its complete continuity established in Section 3, we show the existence of nontrivial solutions of $\left(P_{\lambda}\right)$ for all $\lambda>0$. We will employ the following theorem the so-called global continuation theorem for the proof of our existence theorem.

Let $X$ be a Banach space and $G: \mathbb{R} \times X \rightarrow \mathbb{R}$ completely continuous with $G(0, u)=0$, and consider

$$
u=G(\lambda, u) .
$$

We denote $\mathcal{S}$ as the set of solutions for $(4.1), \mathbb{R}^{+}=[0, \infty)$ and $\mathbb{R}^{-}=(-\infty, 0]$.

Theorem 4.1 (see [5, Theorem 3.2]). Let $X$ be a Banach space and $G: \mathbb{R} \times X \rightarrow X$ continuous and compact and $G(0, u)=0$. Then, $\mathcal{S}$ contains a pair of unbounded components $\mathcal{C}^{+}, \mathcal{C}^{-}$in $\mathbb{R}^{+} \times X$ and $\mathbb{R}^{-} \times X$, respectively, and $\mathcal{C}^{+} \cap \mathcal{C}^{-}=\{(0,0)\}$.

We now state our main existence theorem.

Theorem 4.2. Assume that $h \in \mathscr{d}$ and $f$ holds $(F)$. Then $\left(P_{\lambda}\right)$ has non-trivial solutions for all $\lambda>0$. to solve

Let us take $X=C[0,1]$ with the norm $\|\cdot\|_{\infty}$. We know that, to solve $\left(P_{\lambda}\right)$, it is enough

$$
u=G(\lambda, u),
$$

where $G$ is defined in Section 3. And we have shown that $G$ is continuous and compact in Section 3. Since $G(0, u)=0$ is obvious, employing Theorem 4.1, we obtain an unbounded continuum $\mathrm{C}^{+}$. If we provide a priori boundedness of solutions for $\left(P_{\lambda}\right)$, then the unbounded continuum guarantees the existence of solutions for all $\lambda>0$. Therefore, to complete the proof of Theorem 4.2, we need to give a priori estimate of solutions for $\left(P_{\curlywedge}\right)$.

Lemma 4.3. Assume that $h \in \mathcal{H}$ and $f$ holds $(F)$. Let $(\lambda, u)$ be a solution for $\left(P_{\lambda}\right)$ with $\lambda \in(0, \Lambda]$. Then, there exists a constant $C(\Lambda)$ which depends only on $\Lambda$ such that $\|u\|_{\infty} \leq C(\Lambda)$. 
Proof. Let us define a function $K:(0, \infty) \times \mathbb{R} \rightarrow \mathbb{R}$ which satisfies the following property: $K\left(\lambda_{n}, h\right) \rightarrow 0$ as $\lambda_{n} \rightarrow 0$. Since $h \in \mathscr{H}$, for given $\Lambda>0$, there exists $\varepsilon>0$ such that

$$
\begin{aligned}
\Lambda^{p-1} \max \{ & \int_{0}^{1 / 2} \varphi_{p}^{-1}\left(|K(\varepsilon, h)|+\varepsilon \int_{s}^{1 / 2}|h(r)| d r\right) d s, \\
& \left.\int_{1 / 2}^{1} \varphi_{p}^{-1}\left(|K(\varepsilon, h)|+\varepsilon \int_{1 / 2}^{s}|h(r)| d r\right) d s\right\}<\frac{1}{2} .
\end{aligned}
$$

Then, since $f_{\infty}=0$, for $\varepsilon$ given above, there exists $N>0$ such that for all $s$ with $|s| \geq N$,

$$
|f(s)| \leq \varepsilon|s|^{p-1}
$$

Since $f$ is continuous on $[-N, N]$, for $N$ given above, there exists a maximum value $M>0$ for $|f|$ on $[-N, N]$, that is, for all $s$ with $|s| \leq N$, we have $|f(s)| \leq M$. Let us divide the interval $[0,1]$ into $I$ and $J$ as

$$
\begin{aligned}
& I:=\{t \in[0,1]:|u(t)| \geq N\}, \\
& J:=\{t \in[0,1]:|u(t)| \leq N\},
\end{aligned}
$$

respectively. Then, we can estimate solution $u$ as follows:

$$
\begin{aligned}
|u(t)| & \leq \begin{cases}\int_{0}^{t} \varphi_{p}^{-1}\left(|\alpha(\lambda h f(u))|+\int_{s}^{1 / 2} \lambda|h(r) f(u(r))| d r\right) d s, & 0 \leq t \leq \frac{1}{2}, \\
\int_{t}^{1} \varphi_{p}^{-1}\left(|\alpha(\lambda h f(u))|+\int_{1 / 2}^{s} \lambda|h(r) f(u(r))| d r\right) d s, & \frac{1}{2} \leq t \leq 1,\end{cases} \\
& =\int_{I}+\int_{J},
\end{aligned}
$$

where $\int_{\Gamma}$ with $\Gamma=I$ or $J$ can be given as

$$
\int_{\Gamma}= \begin{cases}\int_{\Gamma} \varphi_{p}^{-1}\left(|\alpha(\lambda h f(u))|+\int_{s}^{1 / 2} \lambda|h(r) f(u(r))| d r\right) d s, & 0 \leq t \leq \frac{1}{2} \\ \int_{\Gamma} \varphi_{p}^{-1}\left(|\alpha(\lambda h f(u))|+\int_{1 / 2}^{s} \lambda|h(r) f(u(r))| d r\right) d s, & \frac{1}{2} \leq t \leq 1 .\end{cases}
$$


By Lemma 3.1, if $|\lambda f(u)| \leq M$ for some $M>0$, then there exists a constant $K(M, h)>0$ such that $|\alpha(\lambda h f(u))| \leq K(M, h)$. Thus, on $J$, we have

$$
\begin{aligned}
& \int_{J}= \begin{cases}\int_{J} \varphi_{p}^{-1}\left(|\alpha(\lambda h f(u))|+\int_{s}^{1 / 2} \lambda|h(r) f(u(r))| d r\right) d s, & 0 \leq t \leq \frac{1}{2}, \\
\int_{J} \varphi_{p}^{-1}\left(|\alpha(\lambda h f(u))|+\int_{1 / 2}^{s} \lambda|h(r) f(u(r))| d r\right) d s, & \frac{1}{2} \leq t \leq 1,\end{cases} \\
& \leq \begin{cases}\int_{J} \varphi_{p}^{-1}\left(|K(\Lambda M, h)|+\Lambda M \int_{s}^{1 / 2}|h(r)| d r\right) d s, & 0 \leq t \leq \frac{1}{2}, \\
\int_{J} \varphi_{p}^{-1}\left(|K(\Lambda M, h)|+\Lambda M \int_{1 / 2}^{s}|h(r)| d r\right) d s, & \frac{1}{2} \leq t \leq 1,\end{cases} \\
& \leq \begin{cases}\int_{0}^{1 / 2} \varphi_{p}^{-1}\left(|K(\Lambda M, h)|+\Lambda M \int_{s}^{1 / 2}|h(r)| d r\right) d s, & 0 \leq t \leq \frac{1}{2} \\
\int_{0}^{1 / 2} \varphi_{p}^{-1}\left(|K(\Lambda M, h)|+\Lambda M \int_{1 / 2}^{s}|h(r)| d r\right) d s, & \frac{1}{2} \leq t \leq 1\end{cases} \\
& \leq K C_{\Lambda}
\end{aligned}
$$

where $C_{\Lambda}=\max \{|K(\Lambda M, h)|, \Lambda M\}$ and

$$
K=\max \left\{\int_{0}^{1 / 2} \varphi_{p}^{-1}\left(1+\int_{s}^{1 / 2}|h(r)| d r\right) d s, \int_{1 / 2}^{1} \varphi_{p}^{-1}\left(1+\int_{1 / 2}^{s}|h(r)| d r\right) d s\right\}
$$

On $I$, using Corollaries 2.4 and 3.3, and $\lambda / \Lambda<1$, we obtain

$$
\begin{aligned}
& \int_{I} \leq\left\{\begin{array}{l}
\int_{I} \varphi_{p}^{-1}\left(\left|\alpha\left(\frac{\lambda}{\Lambda} h \frac{f(u)}{\|u\|_{\infty}^{p-1}}\right)\right|+\int_{s}^{1 / 2} \frac{\lambda}{\Lambda}|h(r)| \frac{f(u)}{\|u\|_{\infty}^{p-1}} \mid d r\right) d s \Lambda^{p-1}\|u\|_{\infty}, \quad 0 \leq t \leq \frac{1}{2}, \\
\int_{I} \varphi_{p}^{-1}\left(\left|\alpha\left(\frac{\lambda}{\Lambda} h \frac{f(u)}{\|u\|_{\infty}^{p-1}}\right)\right|+\int_{1 / 2}^{s} \frac{\lambda}{\Lambda}|h(r)| \frac{f(u)}{\|u\|_{\infty}^{p-1}} \mid d r\right) d s \Lambda^{p-1}\|u\|_{\infty}, \quad \frac{1}{2} \leq t \leq 1,
\end{array}\right. \\
& \leq \begin{cases}\int_{I} \varphi_{p}^{-1}\left(K(\varepsilon, h)+\varepsilon \int_{s}^{1 / 2}|h(r)| d r\right) d s \Lambda^{p-1}\|u\|_{\infty}, & 0 \leq t \leq \frac{1}{2} \\
\int_{I} \varphi_{p}^{-1}\left(K(\varepsilon, h)+\varepsilon \int_{1 / 2}^{s}|h(r)| d r\right) d s \Lambda^{p-1}\|u\|_{\infty}, & \frac{1}{2} \leq t \leq 1,\end{cases} \\
& \leq \begin{cases}\int_{0}^{1 / 2} \varphi_{p}^{-1}\left(K(\varepsilon, h)+\varepsilon \int_{s}^{1 / 2}|h(r)| d r\right) d s \Lambda^{p-1}\|u\|_{\infty}, & 0 \leq t \leq \frac{1}{2} \\
\int_{1 / 2}^{1} \varphi_{p}^{-1}\left(K(\varepsilon, h)+\varepsilon \int_{1 / 2}^{s}|h(r)| d r\right) d s \Lambda^{p-1}\|u\|_{\infty}, & \frac{1}{2} \leq t \leq 1,\end{cases} \\
& \leq \frac{1}{2}\|u\|_{\infty} .
\end{aligned}
$$

Therefore, we have

$$
|u(t)| \leq K C_{\Lambda}+\frac{1}{2}\|u\|_{\infty}
$$


so that

$$
\|u\|_{\infty} \leq 2 K C_{\Lambda}
$$

and this completes the proof.

\section{Acknowledgment}

The authors express their thanks to Professor Yuki Naito for valuable suggestions. The first author was supported by the 2010 Research Fund of the University of Ulsan.

\section{References}

[1] R. Manásevich and J. Mawhin, "Periodic solutions for nonlinear systems with $p$-Laplacian-like operators," Journal of Differential Equations, vol. 145, no. 2, pp. 367-393, 1998.

[2] R. Manásevich and J. Mawhin, "Boundary value problems for nonlinear perturbations of vector $p$ Laplacian-like operators," Journal of the Korean Mathematical Society, vol. 37, no. 5, pp. 665-685, 2000.

[3] R. P. Agarwal, H. Lü, and D. O'Regan, "Eigenvalues and the one-dimensional p-Laplacian," Journal of Mathematical Analysis and Applications, vol. 266, no. 2, pp. 383-400, 2002.

[4] D. Hai, "Positive solutions to a class of elliptic boundary value problems," Journal of Mathematical Analysis and Applications, vol. 227, no. 1, pp. 195-199, 1998.

[5] P. H. Rabinowitz, Contributions to Nonlinear Functional Analysis, Academic Press, New York, NY, USA, 1971. 


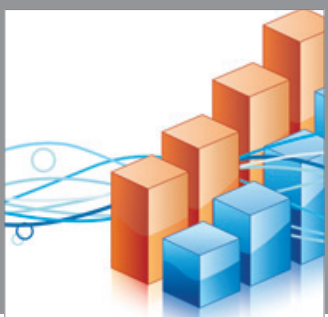

Advances in

Operations Research

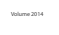

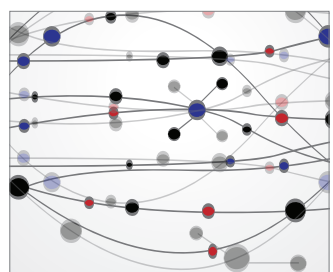

\section{The Scientific} World Journal
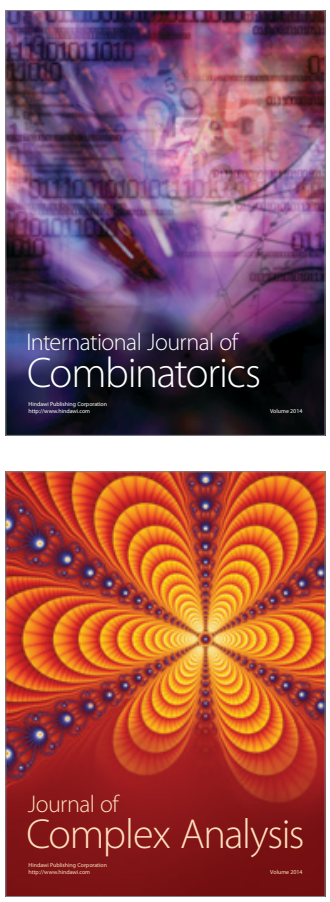

International Journal of

Mathematics and

Mathematical

Sciences
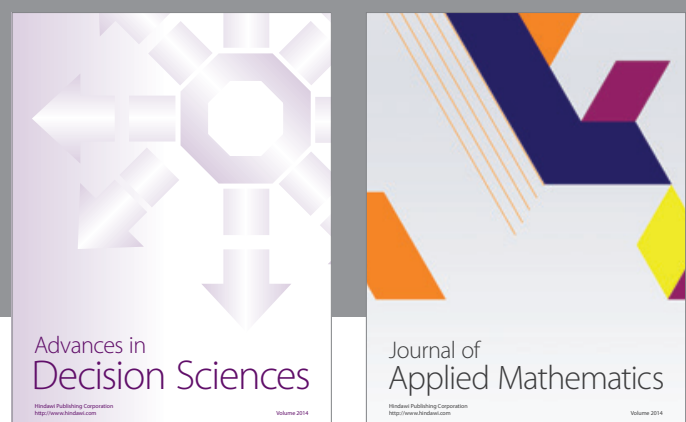

Journal of

Applied Mathematics
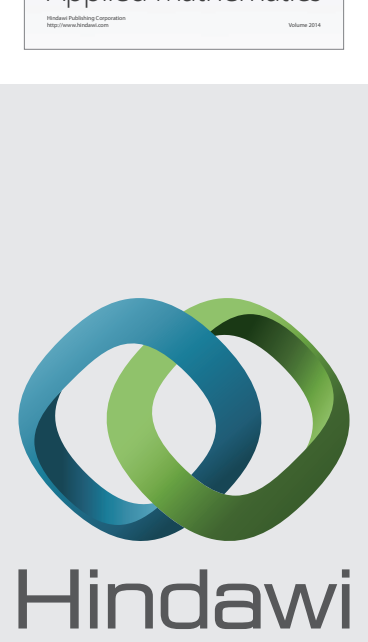

Submit your manuscripts at http://www.hindawi.com
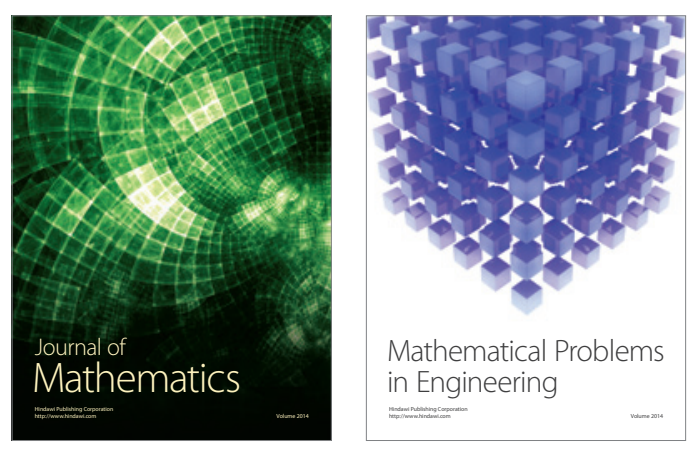

Mathematical Problems in Engineering
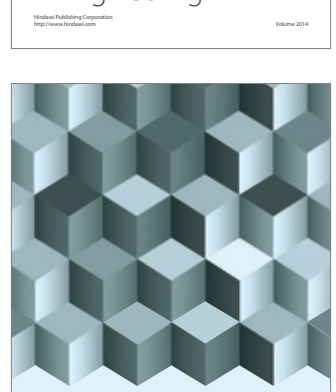

Journal of

Function Spaces
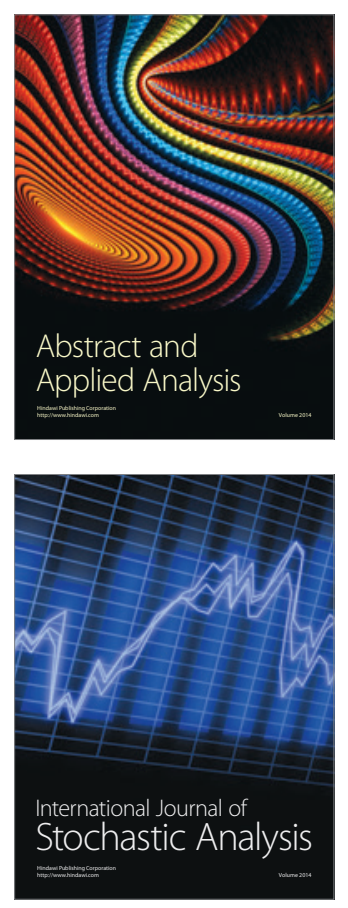

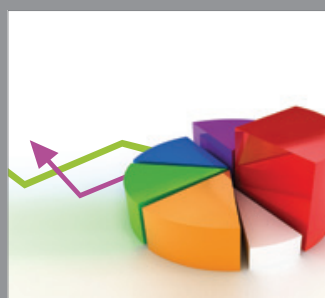

ournal of

Probability and Statistics

Promensencen
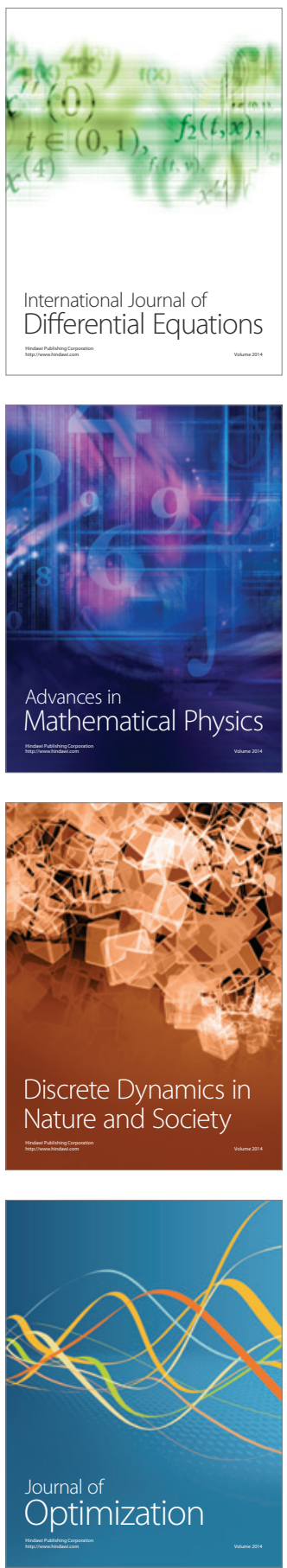